\title{
Linkage of crystal lattice and photodynamic behavior of organic crystals
}

\author{
Nayera Abdelaziz ${ }^{a}$, Jeanette A. Krause ${ }^{b}$ and Anna D. Gudmundsdottir ${ }^{c}$
}

aDepartment of Chemistry, University of Cincinnati, Cincinnati, Ohio 45221-0172, United States

babdelanm@mail.uc.edu

ckrauseje@ucmail.uc.edu

dgudmunad@ucmail.uc.edu

Our group previously reported the first detection of triplet vinylnitrenes in solution by preforming transient spectroscopy of azirine and isoxazole derivatives. We also studied solution photochemistry of 3-azido-1-indenone, which resulted in dimerization of vinylnitrene. Here, we are studying the solid-state photochemistry of 3-azido-1-indenones, which undergoes different photoreacivity from solution due to the rigid structure of the compound in solid state. We are correlating the photoreactivity of both unsubstituted, and substituted 3-azido-1-indenones, to their X-ray crystal structures. Also, we are studying mechanical response of the crystals to light. 